\title{
Síntomas, percepción y demanda de atención en salud mental en niños y adolescentes de la Ciudad de México
}

\author{
Jorge Javier Caraveo-Anduaga, MC, MSP,(1) Eduardo Colmenares-Bermúdez, MC, (1)
} N ora Angélica Martínez-Vélez, Lic Psic., ${ }^{(1)}$

\section{Caraveo-Anduaga JJ, Colmenares-Bermúdez E, Martínez-Vélez NA. \\ Síntomas, percepción y demanda de atención en salud mental en niños y adolescentes de la Ciudad de México. Salud Publica Mex 2002;44:492-498. El texto completo en inglés de este artículo está} disponible en: http://www.insp.mx/salud/index.html

\begin{abstract}
Resumen
Objetivo. Estimar la prevalencia de síntomas emocionales y conductuales en niños y ado lescentes. Identificar las manifestaciones que son percibidas por los padres como necesidades potenciales de atención para sus hijos, y estimar la búsqueda de ser vicios de salud mental. Material y métodos Los datos proceden de una encuesta efectuada de julio a noviembre de 1995 en hogares y fue representativa de las personas entre 18 y 65 años de edad del Distrito Federal, México. Se investigó la presencia de 27 sínto mas en todos y cada uno de los hijos de entre 4 a 16 años de edad que vivían con el adulto entrevistado, así como de la percepción y búsqueda de ayuda para los menores sintomáticos. En el análisis se utilizó la regresión logística. Resultados. Se obtuvo información acerca de 1685 menores. La mitad de la población resultó sintomática. La necesidad de atención fue solamente considerada para $25 \%$, y la búsqueda de atención para $13 \%$. Los síntomas de internalización fueron percibidos más frecuentemente y llevados a la consulta por los padres. Conclusiones. Los resultados sugieren un retraso en la atención. Los datos del estudio serann de utilidad para los clínicos y para la planeación de las políticas y programas de salud mental en los servicios de atención primaria. El texto completo en inglés de este artículo está disponible en: http://www.insp.mx/salud/index.html
\end{abstract}

Palabras clave: prevalencia; servicios salud mental; niño; adolescencia; México

\author{
Caraveo-Anduaga JJ, Colmenares-Bermúdez E, \\ Martínez-Vélez NA. \\ Mental symptoms perceptions of healthcare needs, \\ and health care seeking behaviors, \\ among children and adolescents in Mexico City. \\ Salud Publica Mex 2002;44:492-498. \\ The English version of this paper \\ is available at: http://www.insp.mx/salud/index.html
}

\begin{abstract}
A bstract
Objectives To estimate the prevalence of behavioral and emotional symptoms in children and adolescents; to identify parents' perception of their children's healthcare needs; and to estimate the frequency of seeking mental healthcare services. Material and MethodsA ho usehold survey was carried out between July and N ovember 1995 in Mexico City. The survey was representative of persons aged 18 to 65 years. Study subjects were 1685 children 4 to 16 years of age and living in the same household as their parents. Informant parents provided data on their children regarding the presence of 27 symptoms, their perceptions of healthcare needs of symptomatic children, and mental healthcareseeking behaviors. Lo gistic regression was used for statistical analysis. Results. Symptoms were reported for half of the population. Twenty-five percent of children was perceived by their parents as in need for healthcare, and only 13\% sought healthcare. Internalization symptoms were more frequently perceived by parents and led to seeking healthcare. Conclusions. Study findings suggest a delay in seeking mental healthcare in children and should be of value for clinicians, and to establish primary level mental healthcare policies and programs. The English version of this paper is available at: http://www.insp.mx/salud/index.html
\end{abstract}

Key words: prevalence; mental health services; child; adolescence; Mexico

Este trabajo obtuvo el Segundo lugar del Premio de la Coordinación General de los Institutos N acionales de Salud en el área de Investigaciones en Salud Pública y Ciencias Sociales, octubre 2001.

Esta investigación fue financiada por CO N ACYT, proyecto número 2077- H 9302.

(1) División de Investigaciones Epidemiológicas y Sociales. Instituto N acional de Psiquiatría "Ramón de la Fuente". México, D.F., México.

Fecha de recibido: 10 de agosto de 2001 - Fecha de aprobado: 2 de junio de 2002

Solicitud de sobretiros: Dr. Jorge J Caraveo-Anduaga. Instituto N acional de Psiquiatría. División de Investigaciones Epidemiológicas y Sociales. Calzada México-Xochimilco 101. San Lorenzo Huipulco,Tlalpan 14370. México, D.F., México.

Correo electrónico: caraveoj@ imp.edu.mx 
$\mathrm{E}$ n México, poco más de $40 \%$ de la población es menor de 18 años. ${ }^{1-3}$ La prevalencia de los problemas de salud mental de la población infantil urbana que tiene entre 3 y 12 años se ha estimado en $16 \%$ mediante el Cuestionario de Reporte para Niños (RQC). Así, se ha encontrado que a mayor edad y para ambos sexos, fue superior el reporte de síntomas. ${ }^{4-6}$

Los estudios de la psicopatología del desarrollo han mostrado que algunos problemas y trastornos iniciados en la infancia persisten hasta la edad adulta, y se asocian con otros trastornos psiquiátricos. Durante la adolescencia, otros trastornos favorecen conductas de riesgo y se complican con ellas, como el uso y abuso de sustancias, las conductas suicidas, los delitos y actos violentos, así como los embarazos. ${ }^{7,8}$ Estudios recientes del Consorcio Internacional de Epidemiología Psiquiátrica, que incluye datos de la población adulta de la Ciudad de México, han encontrado que los trastornos de ansiedad de inicio temprano preceden al uso, el abuso y la dependencia de sustancias. Lo anterior pone de relieve la necesidad de identificar cuanto antes los problemas que eventualmente conducen a otras patologías. ${ }^{9,10}$ Sin embargo, a diferencia de los adultos, en el caso de los niños y los adolescentes, la percepción de la necesidad de atención y la búsqueda efectiva dependen precisamente de los primeros, sean éstos los padres $u$ otros familiares responsables, o bien, maestros, médicos o el personal de salud a cargo de los programas de vigilancia de la salud en la comunidad.

De acuerdo con la literatura, los síntomas infantiles por los cuales se acude en busca de ayuda a los servicios de salud mental se han agrupado según sus manifestaciones de externalización, como la hiperactividad, la impulsividad y otros síntomas de conducta desorganizada, reportados como más frecuentes y a los que con mayor premura se acude. A su vez, las manifestaciones de internalización incluyen principalmente las relacionadas con la angustia, los estados alterados del ánimo, y para éstas se reconoce un mayor retraso en la atención. ${ }^{11-14}$

Hasta el momento, no hay estudios en la población general de México acerca de qué manifestaciones motivarían a los padres a solicitar ayuda en salud mental para sus hijos. Lo anterior tiene que ver con la falta de conocimiento que existe en torno a la percepción de la necesidad para atender los problemas de salud mental en la infancia y la adolescencia. Por otra parte, hay evidencias de que la búsqueda de atención se emprende con un retraso considerable; esto es, de un año para los trastornos emocionales y de hasta ocho años para el retraso mental. ${ }^{15-17}$ Por ello, en la práctica médica general y en los programas de vigilancia de la salud en la comunidad, se requieren instrumentos sencillos, confiables y válidos para la detección y el tratamiento oportuno de las alteraciones psicopatológicas. ${ }^{5,6,18}$

El presente estudio tiene los siguientes objetivos: 1) estimar la prevalencia de las manifestaciones sintomatológicas emocionales y conductuales reportadas acerca de menores de entre 4 a 16 años de edad en el Distrito Federal; 2) identificar las manifestaciones que son percibidas por los padres como necesidades potenciales de atención para sus hijos, y 3) estimar la búsqueda de servicios de salud mental que, efectivamente, se ha realizado.

\section{Material y métodos}

Los datos proceden de una encuesta representativa de hogares de personas entre 18 y 65 años de edad que en 1995 vivían de manera permanente o temporal en hogares de las 16 delegaciones políticas del Distrito Federal. ${ }^{19}$ En los hogares donde el adulto entrevistado tenía hijos entre 4 y 16 años de edad se recolectó además información específica para cada uno de ellos. El diseño de la muestra fue polietápico, con estratificación por sexo y según la disponibilidad de servicios de salud mental en las delegaciones. La tasa de respuesta fue de $60.4 \% .{ }^{10}$ La encuesta constó de 13 secciones, cada una de las cuales exploró tanto aspectos específicos de la psicopatología como otras áreas de interés asociadas con las anteriores.

Así, la sección Q investigó de manera específica la presencia de 27 aspectos conductuales y emocionales de todos y cada uno de los hijos de entre 4 a 16 años de edad que vivían con el adulto entrevistado. Los aspectos investigados corresponden a los 10 reactivos del Cuestionario de Reporte para Niños, empleado en la Encuesta Nacional de Salud Mental, ${ }^{4,5}$ así como a otros 17 reactivos seleccionados de la Lista de Conductas en los Niños, de la versión para padres (CBCL-P), de Achenbach, ${ }^{20}$ de manera que la información fuera más precisa para definir casos y tipos de patologías probables. Se preguntó acerca de la presencia de cada síntoma y conducta, resaltando que estas manifestaciones fuesen frecuentes durante el último año, y de su continuidad en el caso de haberse iniciado anteriormente. Al final, se preguntó, en general, la percepción que tenía el adulto entrevistado acerca de la necesidad de ayuda para los menores con síntomas positivos, así como acerca de la búsqueda específica de servicios en caso de haberla llevado a cabo en cada uno de los hijos.

Para el análisis de la información se utilizó el programa estadístico Stata 6.0,* que permite estimar la va-

* STATA 6.0: Statistics/ data analysis STATA Corporation. Texas U.S.A. 
rianza y el error estándar tomando en cuenta el diseño polietápico y estratificado del estudio. Por otro lado, con el fin de identificar las manifestaciones para las cuales se consideraran necesarias la atención y la ayuda en los servicios de salud mental, se realizaron análisis de regresión logística, tomando como variable dependiente la percepción de la necesidad y, como variables independientes o predictoras, los 27 síntomas investigados, el sexo del padre entrevistado, el sexo y la edad de los menores, agrupada en los siguientes intervalos: 4 a 5; 6 a 8; 9 a 12 y de 13 a 16 años. Se tomó en cuenta, asimismo, la estimación del ingreso mensual familiar para 1995, según los criterios de la Asociación Mexicana de Agencias de Investigación de Mercado y Opinión Pública (AMAI): 1000 pesos o menos; de 1000 a 1 999; de 2000 a 3 999; de 4000 a 12999 y de 13000 o más.

Un revisor anónimo opinó acerca de la importancia de considerar el posible agrupamiento de observaciones en los casos en que hubiera varios hermanos en una misma familia entrevistada. Al respecto, conviene aclarar que por medio de ecuaciones de estimación generalizada (generalized estimating equations), ${ }^{21}$ se realizaron análisis capaces de acomodar la interdependencia de observaciones al interior de un mismo grupo familiar. Puesto que no se encontraron diferencias sustantivas, en aras de la brevedad, reportamos sólo los resultados de los modelos más sencillos que se consideran en el diseño polietápico y estratificado del estudio.

\section{Resultados}

En el cuadro I se presentan las características sociodemográficas de la muestra poblacional incluida en este estudio. De los 1932 adultos entrevistados, 924 tenían hijos entre 4 y 16 años de edad, que vivían en el mismo hogar. Así, 380 padres y 544 madres brindaron información acerca de un total de 1686 menores que representan a 1896805 habitantes de la Ciudad de México. Considerando la naturaleza dicotómica de los 27 reactivos, la confiabilidad del instrumento se midió por medio del coeficiente de Kuder-Richardson (KR-20), con el que se obtuvo un valor de 0.81 , en un margen de 0.76 a 0.85 .

De acuerdo con la puntuación global del cuestionario, $48.6 \%(n=820)$ de la población de niños y adolescentes resultó asintomática; $17 \%$ reportó la presencia de un solo síntoma; $12 \%$ presentaron dos; en $6 \%$ se reportaron tres; en $5 \%$, cuatro; en 3\%, cinco; en $2 \%$ se reportaron seis y siete. En general, en 16\% se reportaron cuatro o más síntomas.

\section{Cuadro I \\ Características sociodemográficas de la muestra, Distrito Federal, México, 1995}

\begin{tabular}{lllllll} 
& \multicolumn{2}{c}{ Hombres } & & \multicolumn{2}{c}{ Mujeres } \\
\cline { 2 - 3 } A dultos con hijos de 4 a 16 años de edad & 380 & 41 & & 544 & 59
\end{tabular}

Menores, grupos de edad (años)

\begin{tabular}{lrrrr}
4 a 5 & 141 & 8.4 & 134 & 7.9 \\
\hline 6 a 8 & 230 & 13.6 & 200 & 11.9 \\
\hline 9 a 12 & 261 & 15.5 & 259 & 15.4 \\
\hline 13 a 16 & 233 & 13.8 & 228 & 13.5
\end{tabular}

Ingreso familiar mensual

\begin{tabular}{lrrrr} 
de \$ 13000 o más & 22 & 1.3 & 23 & 1.4 \\
\hline de \$ 4000 a 12 999 & 114 & 6.8 & 100 & 5.9 \\
\hline de \$ 2000 a 3999 & 211 & 12.5 & 223 & 13.2 \\
\hline de \$ 1000 a 1999 & 235 & 13.9 & 231 & 13.7 \\
\hline de \$ 1000 o menos & 269 & 16 & 225 & 13.3 \\
\hline N o especificado & 14 & 0.8 & 19 & 1.1
\end{tabular}

En el cuadro II se presenta la prevalencia de las diferentes manifestaciones sintomáticas investigadas. Las siguientes son las que arrojaron una mayor prevalencia: inquietud, $19 \%$; irritabilidad, $17 \%$; nerviosismo, $16 \%$; déficit de la atención, $14 \%$; desobediencia, $13 \%$; explosividad, $11 \%$ y conducta dependiente, $9 \%$. Con excepción de la irritabilidad, todas las demás manifestaciones se reportaron como conductas frecuentes con más de un año de presentación.

De acuerdo con el instrumento, se preguntó al padre o la madre si consideraba que el menor o los menores sintomáticos ameritaran recibir una atención en salud mental. Sólo en $26 \%$ de los casos los padres consideraron que sus hijos requerían ayuda en salud mental. Entre aquellos menores con una puntuación de uno a tres síntomas se consideró necesaria la ayuda sólo para $17 \%$, en tanto que entre los que obtuvieron puntajes entre cuatro o más síntomas, la percepción de necesidad de atención fue de $45 \%$. Aun con esta última cifra, los padres no consideraron la necesidad de ayuda para poco más de la mitad de los menores con mayor probabilidad de cursar con algún tipo de trastorno.

En el cuadro III se presentan los resultados de la regresión logística, ajustados para cada una de las variables del modelo, mostrando la asociación con la percepción de necesidad de la atención. Resalta que ni el nivel socioeconómico del hogar, como tampoco el 


\section{Cuadro II \\ Prevalencia de síntomas en población de 4 a 16 años, Distrito Federal, México, 1995}

\begin{tabular}{|c|c|c|}
\hline Síntomas & Prevalencia (\%) & IC 95\% \\
\hline Inquieto & 19.4 & $16.6-22.5$ \\
\hline Irritable & 17.4 & $14.2-21.1$ \\
\hline N ervioso, miedoso & 16.4 & $13.3-20.2$ \\
\hline Distraído & 13.7 & $11.9-15.7$ \\
\hline Desobediente, peleonero & 12.7 & $10.4-15.4$ \\
\hline Explosivo & 10.9 & $8.9-13.3$ \\
\hline Dependiente & 9.3 & $7.5-11.3$ \\
\hline Lento aprendizaje & 7.4 & $5.9-9.1$ \\
\hline Sobrepeso & 7.2 & $5.9-8.8$ \\
\hline Bajo peso & 7.1 & $5.8-8.6$ \\
\hline Triste, deprimido & 6.3 & $5.1-7.7$ \\
\hline Mentiroso & 5.9 & $4.4-7.8$ \\
\hline No trabaja en la escuela & 5.2 & $4.2-6.4$ \\
\hline C efaleas frecuentes & 5.1 & $3.8-6.7$ \\
\hline Lenguaje anormal & 4.9 & $3.3-7.3$ \\
\hline Malas compañías & 4.0 & $2.9-5.4$ \\
\hline Enuresis* & 3.8 & $2.7-5.3$ \\
\hline Dolores físicos ${ }^{\ddagger}$ & 3.6 & $2.7-4.7$ \\
\hline Pesadillas frecuentes & 2.9 & $1.9-4.4$ \\
\hline En babia, mirando al vacío & 2.8 & $1.9-4.1$ \\
\hline Aislado & 2.2 & $1.4-3.3$ \\
\hline Problemas para dormir & 2.0 & $1.2-3.2$ \\
\hline Miedo a ir a la escuela & 1.5 & $0.9-2.4$ \\
\hline Ataques, convulsiones & 1.4 & $0.8-2.5$ \\
\hline Roba & 1.0 & $0.5-1.9$ \\
\hline Fugas del hogar & 0.9 & $0.5-1.6$ \\
\hline Usa alcohol o drogas ${ }^{\S}$ & 0.5 & $0.1-2.2$ \\
\hline \multicolumn{3}{|l|}{$\begin{array}{l}\S \text { sin problema médico } 13 \text { a } 16 \text { años } \\
\S \text { de }\end{array}$} \\
\hline$(n=1686)$ & & \\
\hline
\end{tabular}

sexo del informante se encontraron asociados estadísticamente con la necesidad de atención para las conductas reportadas acerca de los hijos. Por otra parte, en relación con el sexo y el grupo de edad de los menores, la percepción de necesidad de atención resultó significativa sólo para los escolares de 9 a 12 años de uno u otro sexo. En otras palabras, el riesgo de ser percibido como niño o niña con problemas se incrementa para este grupo de edad. Respecto a los síntomas, dos de ellos, "nervioso, miedoso" e "irritable, geniudo" , corresponden a características con alta prevalencia, en tanto que los otros cuatro fueron síntomas poco frecuentes pero de mayor importancia clínica, $y$, a su vez, mostraron una asociación mayor en términos de la razón de momios, con la percepción de necesidad de atención.
El análisis de regresión logística mostró que, en efecto, se buscó ayuda para la mayoría de los síntomas percibidos como necesidades, a los que se agregaron otras manifestaciones, como el "lento aprendizaje" y el "lenguaje anormal" (cuadro IV).

\section{Cuadro III \\ SíNTOMAS Y CARACTERÍSTICAS DE LOS MENORES ASOCIADOS* CON LA PERCEPCIÓN DE NECESIDAD de ayuda, Distrito Federal, México, 1995}

\begin{tabular}{lll} 
Síntomas & RM & IC $95 \%$ \\
N ervioso, miedoso & 1.8 & $1.0-3.1$ \\
\hline Enuresis & 2.1 & $1.3-3.3$ \\
\hline Aislado & 3.9 & $1.7-8.9$ \\
\hline Irritable, geniudo & 2.1 & $1.4-3.1$ \\
\hline En babia, mirando al vacío & 2.5 & $1.1-5.8$ \\
\hline Ataques, convulsiones & 3.4 & $1.1-10.3$ \\
\hline Niños de 9 a 12 años & 2.5 & $1.1-5.7$ \\
\hline$N$ iñas de 9 a 12 años & 3.4 & $1.3-9.3$
\end{tabular}

Ajustando simultáneamente por todos los síntomas, sexo del informante, grupos de edad y sexo de los menores e ingreso familiar estimado, y considerando el diseño polietápico y estratificado del estudio. Para el análisis se incluyeron sólo a los sujetos con síntomas

$(n=866)^{*}$

* Se presentan solamente las variables con asociaciones estadísticamente significativas.Ver texto

\section{Cuadro IV \\ Síntomas EN LOS MENORES ASOCiAdos CON LA BÚSQUEDA DE AYUDA, Distrito Federal, México, 1995}

\begin{tabular}{lll} 
Síntomas & RM & IC 95\% \\
Lenguaje anormal & 8.6 & $2.5-29.4$ \\
\hline Nervioso, miedoso & 5.0 & $1.9-13.3$ \\
\hline Lento aprendizaje & 3.5 & $1.4-8.8$ \\
\hline Enuresis & 5.0 & $1.3-18.8$ \\
\hline Mentiroso & 4.6 & $1.4-15.2$ \\
\hline Ataques, convulsiones & 6.0 & $1.4-26.4$ \\
\hline En babia, mirando al vacío & 9.0 & $2.4-33.9$
\end{tabular}

Ajustando simultáneamente por todos los síntomas, sexo del informante, grupos de edad y sexo de los menores e ingreso familiar estimado, y considerando el diseño polietápico y estratificado del estudio.Para el análisis se incluyeron sólo a los sujetos para quienes se buscó ayuda

$(n=114)$

* Se presentan solamente las variables con asociaciones estadísticamente significativas.Ver texto 
Sólo 13\% de los posibles casos acudió a solicitar ayuda. De éstos, $80 \%$ acudió con un solo profesional; $13 \%$, con dos; y $6 \%$ con tres o más. Específicamente, $66 \%$ buscaron atención con psicólogos; $28 \%$, con médicos generales; $18 \%$, con trabajadores sociales; $9 \%$, con psiquiatras; $2 \%$, con otros médicos, y $4 \%$ con otros prestadores de servicios.

\section{Discusión}

El presente estudio nos ha mostrado que la mitad de la población de niños y adolescentes entre 4 y 16 años de edad presenta al menos una conducta sintomática de las incluidas en el cuestionario. No obstante, y corroborando estimaciones de estudios anteriores, $16 \%$ presenta mayores probabilidades de cursar con un trastorno. ${ }^{5,6}$ De acuerdo con la percepción de los padres, la necesidad de atención para estos menores se consideró nada más para una cuarta parte de ellos, y en menos de la mitad para los más claramente sintomáticos. La búsqueda de atención fue todavía más reducida -apenas $13 \%$ - y los profesionales más consultados fueron los psicólogos.

Una limitación del estudio es que no contó con la posibilidad de una valoración clínica y, por lo tanto, no es posible señalar si efectivamente los niños y jóvenes con mayor número de síntomas presentaron alteraciones clínicamente importantes. No obstante, al utilizar solamente las 10 preguntas del RQC, a partir de la presencia de dos síntomas positivos, se obtuvo un valor predictivo positivo de $76 \%$ y un valor predictivo negativo de $99 \% .{ }^{6,18}$ De lo anterior se desprende que las estimaciones del presente estudio sean probablemente comparables con respecto a estudios previos. Otra limitación surge de que la pregunta relativa a la percepción de la necesidad de atención se formuló para el conjunto de síntomas reportados y no para cada uno, y tampoco se registró la edad de aparición de los síntomas en aquellos con más de un año de evolución.

Respecto al grupo de edad, de 9 a 12 años, y que es para el que más se consideró la necesidad de atención, en Hong Kong la media de edad de los menores atendidos en una clínica especializada fue de 9.7 años, y en Ankara, de 8.7 años. ${ }^{22,23}$ Por otra parte, de acuerdo con los resultados de un estudio epidemiológico realizado en cuatro comunidades de Estados Unidos de América, Leaf y colaboradores ${ }^{24}$ señalaron que parece existir un importante impedimento para recibir servicios especializados en salud mental, así como la posibilidad de una falta de reconocimiento temprano de las manifestaciones en niños y adolescentes por parte de los padres y de los primeros prestadores de servicios. Los hallazgos del presente estudio concuer- dan con estos resultados y observaciones, y aportan por primera vez información acerca de la percepción de la necesidad de ayuda en relación con síntomas y conductas específicas en comunidad, aspecto que apenas se ha abordado en otras investigaciones en la literatura. ${ }^{25}$

$\mathrm{Al}$ respecto, años atrás resaltamos la utilidad del modelo de Goldberg y Huxley para comprender el proceso que siguen los sujetos para la atención de trastornos mentales. Dicho modelo describe cinco niveles, que van desde la comunidad hasta los servicios de hospitalización, y cuatro filtros que incluyen la conducta del enfermo, la detección del trastorno, la canalización con el especialista y la admisión hospitalaria. Para el primer filtro, el individuo clave es el paciente, y los factores que operan para que acuda a la consulta médica general son el tipo y gravedad de los síntomas, el estrés psicosocial, las actitudes de los familiares, la disponibilidad de los servicios y la accesibilidad económica para el tratamiento. Para el segundo y tercer filtros, el individuo clave es el médico de primer contacto, y los factores que operan para la detección del trastorno incluyen las técnicas de entrevista, el entrenamiento y las actitudes, el patrón de síntomas presentado y las características sociodemográficas del paciente. Los hallazgos de ese estudio señalaron que, desafortunadamente, la cronicidad y el patrón sintomatológico eran las características relevantes en la identificación de los trastornos psiquiátricos por parte del médico general, lo que implicaba un retraso en la atención. 26,27

De acuerdo con los resultados del presente estudio, resalta que ni el informante -el padre o la madre-, ni el nivel socioeconómico del hogar se asociaron positivamente con la percepción de la necesidad de ayuda para los hijos sintomáticos. Estos hallazgos sugieren que, en general, existe probablemente un desconocimiento de la importancia de algunas manifestaciones psicopatológicas que se presentan en la infancia y la adolescencia. Por ejemplo, llama la atención que los síntomas clave del trastorno por déficit de atención e hiperactividad, que estuvieron entre las manifestaciones más frecuentes y que además aparecen tempranamente en el curso de la vida, no se hayan percibido aparentemente como motivos de atención y, menos aún, llevados a consulta. Solamente el bajo rendimiento escolar, uno de los efectos de la atención deficiente pero no exclusivo de ésta, fue objeto de referencia.

Estos hallazgos difieren de lo que se halla en la literatura, principalmente la anglosajona, en la que los menores con síntomas de externalización y trastornos de la conducta predominan sobre aquellos con trastornos emocionales entre la población atendida en los 
servicios clínicos. ${ }^{12-14}$ Por tanto, estos resultados indicarían que posiblemente exista en nuestra población una mayor tolerancia hacia las conductas de externalización, a la vez que un amplio desconocimiento de la importancia de este tipo de conductas como manifestaciones de problemas que afectan la salud mental de los menores, en tanto que los síntomas de internalización, como el aislamiento y el nerviosismo, sí llaman la atención de los adultos. Al respecto, hay evidencia de que en Jamaica y Puerto Rico se alcanzaron puntajes superiores en las escalas de internalización en comparación con las de externalización, lo cual sugiere que hay diferencias, tal vez determinadas culturalmente, en la percepción de síntomas en los menores. ${ }^{28} \mathrm{Si}$, como señalan algunos autores, ${ }^{29}$ el patrón de referencia implica que las necesidades del adulto, más que las del menor, constituyen los determinantes para la búsqueda de atención, la variante observada entre nuestra población plantearía ciertos interrogantes. ¿Cómo se interpretan los síntomas en el contexto de la comunidad? ¿Se presentan acaso en el nivel de la consulta médica de los menores, pero no son detectados por los profesionales? El quehacer de estos últimos y la vigilancia del estado de salud mental de los menores, ¿se verían beneficiados con la utilización del instrumento de tamizaje?

Algunas hipótesis relacionadas con este comportamiento podrían ser, por ejemplo, que el malestar podría sentirse, expresarse y, con el tiempo, convertirse en una necesidad de ayuda para el adulto que convive con menores con problemas de conducta. Asimismo, los problemas de atención y conducta de los menores se pueden identificar equivocadamente como problemas de disciplina y, consecuentemente, ser objeto de diversas medidas "correctivas" que podrían devenir maltrato y complicar el problema.

En conclusión, los resultados del presente estudio indican que hay una menor percepción de la necesidad de atención en salud mental para los menores, y que la búsqueda formal de ayuda es todavía más escasa. Es indudable que en derredor de este tema se requieren estudios cuyos resultados indiquen los obstáculos y sugieran los facilitadores para la detección y atención oportunas. Asimismo, este aspecto acentúa la necesidad de la investigación y el desarrollo de programas para la vigilancia de la salud mental durante la infancia y la adolescencia en nuestro medio. Consideramos que los datos aportados por el presente trabajo serán de utilidad tanto para los clínicos como para los responsables de la formulación de políticas y de la planeación de los programas de los servicios de salud.

\section{Referencias}

1. Instituto $\mathrm{N}$ acional de Estadística, Geografía e Informática. Conteo de Población y Vivienda 1995. México, D.F.: IN EGI, 1996.

2. Instituto Nacional de Estadística, Geografía e Informática. XI Censo General de Población y Vivienda, 1990. Los jóvenes en México. México, D.F.: IN EGI, 1993.

3. Instituto $\mathrm{N}$ acional de Estadística, G eografía e Informática. Los niños en México, XI Censo General de Población y Vivienda, 1990. México, D.F.: IN EGI, 1993.

4. Giel R, D e A rango MV, Climent CE, Harding TW, Ibrahim HHA, Ladrido-Ignacio $L$ et al. Childhood mental disorders in primary health care: Results of observations in four developing countries. Pediatrics 1981;68(5): 677-683.

5. Caraveo AJ, Medina-Mora ME,Tapia CR, Rascón ML, Gómez M,Villatoro J.Trastornos psiquiátricos en niños de la República Mexicana. Resultados de una encuesta de hogares. Psiquiatria 1993:9:137-151.

6. Caraveo AJ, Medina-Mora ME,Villatoro J, López-Lugo EK, Martínez VA. Detección de problemas de salud mental en la infancia. Salud Publica Mex 1995;37(5):445-451.

7. Rutter M. Epidemiological approaches to develo pmental psychopathology. Arch Gen Psychiatry 1988;45:486-495.

8. Hofstra MB, der Ende JV,Verhulst FC. C ontinuity and change of psychopathology from childhood into adulthood:A 14-year follow-up study. J Am Acad Child Adolesc Psychiatry 2000;39(7):850-858.

9. Merikangas KR, Mehta RL, Molnar BE,W alters EE, Swendsen JD,A guilar$G$ axiola $S$ et al. Comorbidity of susbtance use disorders with mood and anxiety disorders: Results of the International Consortium in Psychiatric Epidemiology. Addict Behav 1998;23(6):893-907.

10. World Health O rganization. International Consortium in Psychiatric Epidemiology. Cross-national comparisons of the prevalences and correlates of mental disorders. Bull W orld Health 0 rgan 2000; 78(4):413-426. 11. Rutter M, Cox A, Tupling C, Berger M,Yule W. Attainment and adjustment in two geographical areas: I.The prevalence of psychiatric disorder. $\mathrm{Br}$ J Psychiatry 1975;126:493-509.

12. G arralda ME, Bailey D. C hild and family factors associated with referral to child psychiatrics. Br J Psychiatry 1988;153:81-89.

13.W eisz JR,W eiss B. Studying the "referability" of child clinical problems. J Consult Clin Psychol 1991;59:266-273.

14. $O$ llendick TH, Yang B, King N J, D ong Q , A kande A. Fears in A merican, Australian, $\mathrm{Chinese}$, and $\mathrm{N}$ igerian children and adolescents: A cross-cultural study. J Child Psychol Psychiatry 1996;37:213-220.

15. Caraveo AJ, Mas CC. Necesidades de la población y desarrollo de servicios de salud mental. Salud Publica Mex 1990;32:523-531.

16. De la Fuente R, Medina-Mora ME, Caraveo AJ. La salud mental en México. México, D.F.: Fondo de Cultura Económica, Instituto Mexicano de Psiquiatría, 1997.

17. Gater R, D eA Imeida E, So usa B, Barrientos G, C araveo J,C handrashekar $C R$ et al.The pathways to psychiatric care:A cross-cultural study. Psychol Med 1991;21:761-774.

18. Gómez EM, Rico DH, Caraveo AJ, Guerrero CG.Validez de un instrumento de tamizaje (RQ C).An Inst Mex Psiquiatria 1993;4:204-208.

19. C araveo AJ, Martínez N, Rivera E. Un modelo para estudios epidemiológicos sobre la salud mental y la morbilidad psiquiátrica. Salud Mental 1998;21(1):48-57.

20. A chenbach TM, Edelbrock C. Manual for the child behavior cheklist and revised child behavior profile. Burlington (VT): University of Vermont, 1983.

21. Liang KY, Zeger SL. Longitudinal data analysis using generalized linear models. Biometrika 1986;73:13-22. 
22. H O TP, C hung SY. H elp-seeking behaviors among child psychiatry clinic attenders in Hong Kong. Soc Psychiatry Psychiatr Epidemiol 1996;31: 292-298.

23. Kilic C, Rezaki M, Ustun TB, Gater RA. Pathways to psychiatric care in Ankara. Soc Psychiatry Psychiatr Epidemiol 1994;29:131-136.

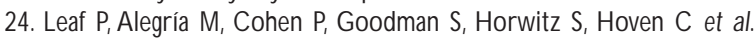
Mental Health service use in the community and schools: Results from four-community MECA study. J Acad Child Adolesc Psychiatry 1996; 35(7):889-897.

25. McKelvey RS, Baldassar LV, Sang D L, Roberts L. Vietnamese parental perceptions of child and adolescent mental illness. J A cad Child Adolesc Psychiatry 1999;38(10):1302-1309.

26. Goldberg D P, Huxley P. Mental illness in the community. The pathway to psychiatric care. London:Tavistock Publications, 1980.
27. Caraveo AJ, González FC, Ramos LL, Mendoza BP. N ecesidades y demandas de atención en los servicios de salud mental. Salud Publica Mex 1986;28(5):504-514.

28. C rijnen AM,A chenbach TM,Verhulst FC. C omparisons of problems reported by parents of children in 12 cultures:Total problems, externalizing and internalizing. J Acad Child Adolesc Psychiatry 1997;36(9): 1269-1277.

29. Target M, Fonagy P.The psychological treatment of child and adolescent psychiatric disorders. En:Roth A, Fonagy P, ed. W hat works for whom?: A critical review of psychotherapy research. N ueva York: The Guilford Press, 1996:263-272 